\title{
Helicity, Reconnection, and Dynamo Effects
}

\author{
Hantao Ji \\ Princeton Plasma Physics Laboratory, Princeton University \\ Princeton, New Jersey
}

\begin{abstract}
The inter-relationships between magnetic helicity, magnetic reconnection, and dynamo effects are discussed. In laboratory experiments, where two plasmas are driven to merge, the helicity content of each plasma strongly affects the reconnection rate as well as the shape of the diffusion region. Conversely, magnetic reconnection events also strongly affect the global helicity, resulting in efficient helicity cancellation (but not dissipation) during counter-helicity reconnection and a finite helicity increase or decrease (but less efficiently than dissipation of magnetic energy) during co-helicity reconnection. Close relationships also exist between magnetic helicity and dynamo effects. The turbulent electromotive force along the mean magnetic field ( $\alpha$-effect), due to either electrostatic turbulence or the electron diamagnetic effect, transports mean-field helicity across space without dissipation. This has been supported by direct measurements of helicity flux in a laboratory plasma. When the dynamo effect is driven by electromagnetic turbulence, helicity in the turbulent field is converted to mean-field helicity. In all cases, however, dynamo processes conserve total helicity except for a small battery effect, consistent with the observation that the helicity is approximately conserved during magnetic relaxation.
\end{abstract}




\section{INTRODUCTION}

Magnetic helicity, a measure of the "knottedness" and the "twistedness" of magnetic field [Woltjer, 1958], is closely related to field line topology [Berger and Field, 1984]. It is defined by

$$
K=\int \mathbf{A} \cdot \mathbf{B} d V
$$

where $\mathbf{A}$ is the vector potential of the magnetic field $\mathbf{B}$ and the integration is over a volume $V$. The magnetic helicity is an invariant within a flux tube in a perfectly conducting plasma. Taylor [1974] conjectured that in a "slightly" resistive plasma the total helicity is wellconserved during plasma relaxation in which the magnetic energy decays toward a minimum-energy state. This well-known hypothesis has been successful [Taylor, 1986] in explaining magnetic structures in laboratory toroidal plasmas, such as the reversed-field-pinch (RFP), spheromak, and multipinch. Recently, there has been growing awareness that the global magnetic helicity contained in flux tubes also plays an important role in solar flare evolution [Berger, 1984; Rust, 1994].

As an elementary process in resistive plasmas, magnetic reconnection [Vasyliunas, 1975; Biskamp, 1993] has long been regarded as a key mechanism in deciding the dynamics of solar flares [e.g., Parker, 1979], magnetospheric substorms [e.g., Akasofu, 1972], and relaxation phenomena in laboratory plasmas [e.g., Taylor, 1974]. Although this is a localized process, it often causes fundamental changes in macroscopic magnetic field topology through cutting and rejoining of field lines. Therefore, an inherent relationship between global helicity and local reconnection events must exist.

Dynamo effects also have been another focal point of research in electrically conductive fluids or plasmas attempting to explain the observed solar and planetary magnetic fields. In particular, generation of an electromotive force (EMF) along a mean field by turbulence, or the well-known $\alpha$-effect [Parker, 1955], is an essential process in amplifying large-scale magnetic fields [e.g., Proctor and Gilbert, 1994]. These dynamo effects drive parallel current which twists up the field lines thus creating magnetic helicity on large scales. Therefore, the helicity also must be closely related to dynamo effects.

In the following sections, we discuss the relationship between magnetic helicity and magnetic reconnection with emphasis on results from recent labora- tory experiments, followed by discussions on the relationships between magnetic helicity and dynamo effects in a plasma.

\section{RELATIONSHIP BETWEEN HELICITY AND RECONNECTION}

\section{1. "Push" and "Pull" Reconnection}

Consider the situation where two plasmas with parallel toroidal current interact with each other. Reconnection of the poloidal fields results in the formation of three regions in the system: private regions associated with each plasma and a public region surrounding both plasmas, as illustrated in Fig. 1 (a). This configuration has been created in a laboratory experiment, MRX (Magnetic Reconnection Experiment) [Yamada et al., 1997], where two internal coils are inserted in each private region (denoted by the closed circles in Fig. 1). After the plasma is formed, the private flux can be further increased to generate "push" reconnection as shown in Fig. 1(b), where the field lines are reconnected from the private regions to public region. On the other hand, when the private flux is decreased the poloidal flux is "pulled" back from the public region to the private regions by reconnection, as shown in Fig. 1(c). Both types of reconnection can occur in nature and can be induced in MRX by changing operational procedures. (Two examples of natural pull reconnection are solar flare and magnetotail reconnection.)

\subsection{Effects of Helicity Content on Reconnection}

The most common description of magnetic field line reconnection is shown in Fig. 2(a), on which the two-dimensional theories have been based [ $\mathrm{Va}$ syliunas, 1975; Biskamp, 1993]. However, magnetic field lines have three vector components and the helicity content of each plasma has a dominate effect on the local reconnection angle, as seen in the threedimensional pictures of Fig. 2(b). When the third component $B_{t}$ vanishes in both plasmas [null-helicity case, Fig. 2(i)], conventional 2-D reconnection is applicable. In the presence of a third component, (1) the field lines reconnect at an angle when uni-directional toroidal fields exist [co-helicity case, Fig. 2(ii)] or (2) they reconnect with anti-parallel geometry when the toroidal fields are oppositely directed [counter-helicity case, Fig. 2(iii)]. Note that the reconnecting field lines are anti-parallel for both null-helicity and counterhelicity cases. 
In MRX, the effect of merging angle on reconnection has been studied extensively [Yamada et al., 1997]. Figure 3 shows examples of time evolution of the poloidal flux contours obtained by a 90-channel 2-D magnetic probe array [Fig. 1(a)] during pull reconnection. Two different shapes of diffusion regions are found, depending on the third components of the reconnecting magnetic fields. Other operational conditions are held constant for each discharge. When no magnetic reconnection is induced, a typical $\mathrm{X}$ shape separatrix region is observed as seen at $t=260$ $\mu \mathrm{s}$ in both Fig. 3(a) and 3(b). As poloidal flux is driven toward the diffusion region, a neutral sheet is formed. Without the third component (null-helicity reconnection), a thin double- $Y$ shaped diffusion region is clearly identified [Fig. 3(a)]. In the presence of an appreciable third component (co-helicity reconnection), an O-shaped sheet current appears [Fig. 3(b)].

The existence of a magnetic island in the co-helicity case indicates a much broader current sheet than the counter-helicity case. Figure 4 presents the radial profiles of poloidal field $B_{Z}$, toroidal field $B_{T}$, toroidal current density $j_{T}$, and pitch of field lines for cohelicity and null-helicity reconnection. In the nullhelicity case, $B_{T}$ is almost zero resulting in an abrupt transition of the pitch of field lines $(\Theta)$ at the reconnection point, while in the co-helicity case, $B_{T}$ is order $B_{Z}$ resulting in a gradual change of $\Theta$ over $R$. In the co-helicity case, the $j_{T}$ profile is broad with width of order $10 \mathrm{~cm}$. In the null-helicity case, one observes a steepening of magnetic field slope at the diffusion region and therefore a sharp neutral sheet current. The thickness of this current sheet is seen to be as narrow as $1 \mathrm{~cm}$, which is found to be proportional to the ion gyro-radius, $\rho_{i}$, defined using the ion temperature at the center and $B_{Z}$ at the knee.

The existence of a sizable toroidal field (co-helicity) also results in a much slower reconnection rate (by a factor of 3 ) than the null-helicity case. Similar observations also have been made in an earlier experiment, where two spheromaks were driven to merge [Yamada et al., 1990; Ono et al., 1993]. A plausible explanation for the observed difference in reconnection rate is based on the incompressibility of the plasma due to the toroidal field pressure in the cohelicity case, while the absence of toroidal field makes the plasma compressible in the null-helicity case [ Ji et al., 1998]. However, a quantitative comparison of the observed reconnection rate with theoretical values is not straightforward, since classical 2-D reconnection models do not explicitly take into account the effects of the third magnetic field component nor plasma compressibility.

\subsection{Effects of Reconnection on Helicity Conservation}

Although magnetic reconnection is a localized process, it often causes topological changes in macroscopic configurations, affecting the globally defined magnetic helicity. Some properties of helicity conservation during magnetic reconnection have been discussed [Wright and Berger, 1991; Pfister and Gekelman, 1991]. In this section, a detailed account of the effects of reconnection on helicity conservation and dissipation of magnetic energy is given.

2.3.1. Time evolution of helicity and energy. The time rate of change of helicity defined by Eq.(1) can be described by

$$
\frac{d K}{d t}=-2 \int \mathbf{E} \cdot \mathbf{B} d V-\int\left(2 \phi \mathbf{B}+\mathbf{A} \times \frac{\partial \mathbf{A}}{\partial t}\right) \cdot d \mathbf{S}
$$

where $\phi$ is the electrostatic potential and $\mathbf{S}$ is the surface surrounding integrated volume. The first term on the right hand side (RHS) represents the volume helicity rate of change while the second and third terms are helicity flux through the surface via inductive and electrostatic means, respectively.

A similar equation can be derived for the time evolution of the volume integrated magnetic energy, $W=\int B^{2} / 2 \mu_{0} d V$,

$$
\frac{d W}{d t}=-\int \mathbf{E} \cdot \mathbf{j} d V-\int(\mathbf{E} \times \mathbf{B}) \cdot d \mathbf{S}
$$

where the first term on the RHS represents the energy dissipation rate and the second term the Poynting flux.

What is of interest here is how much helicity and energy change occurs within the volume of integration during the reconnection process. Therefore, only the volume-dissipative terms $\mathbf{E} \cdot \mathbf{B}$ and $\mathbf{E} \cdot \mathbf{j}$ but not the surface terms (which represent helicity or energy flux) in Eq.(2) and Eq.(3) need to be examined.

2.3.2. Dissipation terms during reconnection. Now consider a Sweet-Parker type of reconnection [Sweet, 1958; Parker, 1957] as illustrated in Fig. 5, where a rectangular diffusion region forms with width $2 \delta$ and length $2 L$. Recently, this type of reconnection has been verified experimentally in MRX, where some other effects, including an enhanced resistivity over the Spitzer value, are taken into account [Ji et al., 1998]. (On the other hand, an alternative 
model by Petschek [1964], which is based on a much smaller diffusion region and standing shock waves, has not yet been confirmed experimentally.) As is typical for magnetic reconnection, the region outside of the diffusion region can be treated by ideal MHD, where $\mathbf{E}+\mathbf{V} \times \mathbf{B}=0$ holds. Therefore,

$$
\begin{aligned}
\mathbf{E} \cdot \mathbf{B} & =E_{T} B_{T}+E_{P} B_{P}=0 \\
\mathbf{E} \cdot \mathbf{j} & =(\mathbf{j} \times \mathbf{B}) \cdot \mathbf{V}
\end{aligned}
$$

leading to no changes in helicity $\left(E_{T} B_{T}\right.$ balances with $-E_{P} B_{P}$ ) and no magnetic energy dissipation (except for an exchange with mechanical energy).

The situation changes when the diffusion region is considered. By definition, the reconnecting field $B_{P} \approx 0$ and the Ohm's law can be approximated by $\mathbf{E}+\mathbf{V} \times \mathbf{B}=\eta^{*} \mathbf{j}$, where $\eta^{*}$ is an effective resistivity which includes all non-ideal MHD effects. Because both $E_{T}$ and the non-reconnecting $B_{T}$ are unchanged from outside the diffusion region, we have

$$
\begin{aligned}
\mathbf{E} \cdot \mathbf{B} & \approx E_{T} B_{T}\left(=\eta^{*} j_{T} B_{T}\right) \\
\mathbf{E} \cdot \mathbf{j} & =(\mathbf{j} \times \mathbf{B}) \cdot \mathbf{V}+\eta^{*} j^{2}
\end{aligned}
$$

leading to possibly non-zero $\mathbf{E} \cdot \mathbf{B}$ (a net change in helicity) and a net energy dissipation. We shall discuss these terms in the following sections for both counterand co-helicity reconnection.

2.3.3. Helicity neutralization during counterhelicity reconnection. Consider the case of counterhelicity reconnection shown in Fig. 2(iii), where the third component $B_{T}$ changes sign across the current sheet. In this case, $B_{T}$ is also reconnected as a result of field line diffusion. Therefore, $B_{T} \approx 0$ in the diffusion region, resulting in no helicity dissipation [Eq.(4)]. However, the original helicity contained in each plasma has been lost as a result of reconnection, and this process can be described as helicity neutralization or cancellation. The end product of the counter-helicity reconnection is a toroidal configuration (called a Field Reversed Configuration, or FRC) consisting of only toroidal current (or poloidal field) with no toroidal field, or no helicity. This physical process has been demonstrated experimentally [ $\mathrm{Ya}$ mada et al., 1990; Ono et al., 1993] as illustrated in Fig. 6. One unique feature of an FRC is that a high $\beta(\sim 1)$ plasma heated by reconnection is confined by the perpendicularly flowing current, most of which is carried by ions. The observed stability of such a configuration cannot be explained by Taylor's theory since it contains no apparent magnetic helicity. Recently, there have been attempts [Steinhaurer and Ishida, 1997; Hegna, 1998] to generalize relaxation theories, using electron helicity to describe parallel current and ion helicity to describe perpendicular current or plasma flow, in order to minimize the total energy including kinetic and thermal energies. Then, FRC plasmas can be classified as a minimum energy state with zero electron helicity (or magnetic helicity) but finite ion helicity (or kinetic helicity) [Steinhaurer and Ishida, 1997].

2.3.4. Helicity change during co-helicity reconnection. As illustrated in Fig. 2(ii), the third component $B_{T}$ will be non-zero during co-helicity reconnection, introducing a non-zero helicity change. With respect to $\mathbf{B}_{T}$, however, the direction of $\mathbf{E}_{T}$ can be parallel or anti-parallel, depending on push or pull reconnection, as discussed previously. For the case of positive helicity as shown in Fig. $7, \mathbf{E}_{T}$ is parallel to $\mathbf{B}_{T}$ during pull reconnection resulting in a negative $-\mathbf{E} \cdot \mathbf{B}$ or decrease in helicity, while $\mathbf{E}_{T}$ is anti-parallel to $\mathbf{B}_{T}$ during push reconnection resulting in a positive $\mathbf{- E} \cdot \mathbf{B}$ or increase in helicity.

An intuitive picture of helicity change during cohelicity reconnection is given as follows. In the case of co-helicity reconnection, only the poloidal field $B_{P}$ is diffused and reconnected in the diffusion region. The poloidal field lines slip from up-stream to downstream as soon as it enters the diffusion region, with a speed on the order of $c$. Meanwhile, the toroidal field $B_{T}$ is not diffused and still frozen with plasma, which moves with a speed on the order of the Alfvén speed $\left(V_{A} \ll c\right)$. This slippage of $B_{P}$ relative to $B_{T}$ gives rise a change in linkage (or helicity) with toroidal flux contained in the diffusion region. Following this argument, two more examples for helicity change can be given as shown in Fig. 8, which are often seen in sawtooth reconnection in tokamaks [ $\mathrm{Na}$ gayama et al., 1991] and relaxation events in reversed field pinches (RFP's) [Schnack et al., 1985] if one regards the "toroidal field" as the parallel component along a resonant line and the "poloidal field" as the perpendicular reconnecting component.

2.3.5. Relative rate of change in helicity and energy. A quantitative account of helicity change always needs to be compared with energy dissipation since only their relative difference has physical meaning. Volume integration of $\mathbf{E} \cdot \mathbf{B}$ and $\mathbf{E} \cdot \mathbf{j}$ over the diffusion region,

$$
\begin{aligned}
\frac{d K}{d t} & =-2 E_{T} B_{T} 2 \delta 2 L 2 \pi R \\
\frac{d W}{d t} & =-E_{T} j_{T} 2 \delta 2 L 2 \pi R
\end{aligned}
$$


gives changes in helicity and energy per reconnected poloidal flux $\Psi$,

$$
\begin{aligned}
\left|\frac{d K}{d \Psi}\right| & =8 \delta L B_{T}=2 \Phi_{\mathrm{DR}} \\
\frac{d W}{d \Psi} & =-\frac{4 L B_{P}}{\mu_{0}}
\end{aligned}
$$

where $2 \pi R$ is the total length of diffusion region, $\Phi_{\mathrm{DR}}$ is toroidal flux contained with the diffusion region. (To derive Eq.(6) and Eq.(7), the relations $d \Psi / d t=$ $2 \pi R E_{T}$ and $j_{T} \delta=B_{P} / \mu_{0}$ have been used.) Since generally $W$ and $K$ are related by $W / K \sim 1 /\left(\mu_{0} L\right)$, the ratio of the rates of change is given by

$$
\left|\frac{W}{K} \frac{d K}{d W}\right| \sim 2 \frac{\delta}{L} \frac{\left|B_{T}\right|}{B_{P}} .
$$

We note that not only the current sheet thickness but also the reconnecting angle $\theta=2 \tan ^{-1}\left(B_{P} /\left|B_{T}\right|\right)$ determine the relative rate of change.

An alternative form of Eq.(8) can be written as

$$
\left|\frac{W}{K} \frac{d K}{d W}\right| \sim 2 \frac{\lambda_{\text {global }}}{\lambda_{\text {DR }}},
$$

where $\lambda \equiv \mu_{0} \mathbf{j} \cdot \mathbf{B} / B^{2}$, a parameter often used in the equation $\boldsymbol{\nabla} \times \mathbf{B}=\lambda \mathbf{B}$ to describe the force free state [Taylor, 1974]. When the plasmas are close to their relaxed states, then $\mu_{0} W / K \sim 1 / L \sim \lambda_{\text {global }}$. Similarly, $\lambda$ can also be defined in the diffusion region as $\lambda_{\mathrm{DR}} \equiv \mu_{0} j_{T} / B_{T}$, although it is not clear why the diffusion region has to be in a relaxed state also. But if this is so as suggested by Biskamp [1993], then the relative ratio of change should only be decided by $2 \delta / L$ since $\lambda_{\mathrm{DR}} \sim 1 / \delta$ and $\lambda_{\text {global }} \sim 1 / L$.

2.3.6. How well is helicity conserved during reconnection?. Based on Eq.(8), the relative ratio of change of helicity to energy due to co-helicity reconnection can be estimated. For pull co-helicity reconnection in MRX, as shown in Fig. 3(b), $\delta / L \sim 1 / 2$ and $B_{T} / B_{P} \sim 1$ so that $|(W / K)(d K / d W)| \sim 1$ if the island structure is ignored. Taking into account the presence of the island structure would likely decrease the estimated ratio.

A second case of interest is the RFP plasmas from where the idea of helicity conservation originates. However, the current sheet thickness $\delta$ is not measured in RFP's during a reconnection event. One way to estimate $\delta$ is to relate it to the relative parallel drift parameter defined as $v_{\text {drift }} / v_{\text {th,e }}\left(v_{\text {drift }} \equiv j / e n\right.$ and $v_{\text {th,e }}$ is the electron thermal velocity) by

$$
\delta=\frac{B_{\mathrm{rec}}}{\mu_{0} j_{\|}}=\frac{B_{\mathrm{rec}}}{\mu_{0} e n v_{\mathrm{th}, e}}\left(\frac{v_{\mathrm{drift}}}{v_{\mathrm{th}, e}}\right)^{-1},
$$

where $B_{\text {rec }}$ is the reconnecting field, which is typically the radial field $B_{r}$ in the RFP. The typical drift parameter can be estimated to be on the order of $0.2-0.3$ based on the observation of current carrying fast electrons [Stoneking et al., 1994] or the measured $j_{\|}, n$, and $T_{e}$ [Ji et al., 1994]. Using typical parameters [ $\mathrm{Ji}$, Prager, and Sarff, 1995] in MST (Madison Symmetrical Torus) plasmas as we shall mention in the next section, i.e., $B_{T}=2 \mathrm{kG}, n=1 \times 10^{19} / \mathrm{m}^{3}, T_{e}=100 \mathrm{eV}$, and the plasma radius $a=0.5 \mathrm{~m}$, we have $\delta / a \sim(1.5$ $2.5) \times 10^{-3}$. Using observed $B_{T} / B_{r} \approx 100$ and $\mu_{0} W / K \approx 1 /(0.73 a)$, the relative rate of change of helicity to energy $|(W / K)(d K / d W)| \sim 0.4-0.7$, which is consistent with the observed $\sim 0.4$ during a relaxation event [Ji, Prager, and Sarff, 1995]. This estimate suggests that the helicity conservation is only marginally satisfied in the RFP plasmas.

The last interesting situation is how well helicity is conserved during a solar flare, where reconnection is considered to play a essential role. Again, the current sheet thickness $\delta$ is undetermined observationally. Using Eq.(9) with a drift parameter of 0.2 and the typical parameters $\left(B_{T}=500 \mathrm{G}, n=10^{15} / \mathrm{m}^{3}\right.$, $\left.T_{e}=100 \mathrm{eV}, L=10,000 \mathrm{~km}\right)$, we have $\delta \sim 300 \mathrm{~m}$ and $\delta / L \sim 3 \times 10^{-5}$. If we choose $B_{T} / B_{P} \sim 1$ as one might consider if the plasma is close to a relaxed state, an estimated relative rate of change $|(W / K)(d K / d W)| \sim 6 \times 10^{-5}$ is obtained. Because any adjustment of plasma parameters is unlikely to change this relative rate to a number close to unity, one may conclude that the helicity is indeed conserved relative to energy change in the solar corona.

\section{RELATIONSHIP BETWEEN HELICITY AND DYNAMO EFFECTS}

The dynamo effect, or, generation of magnetic field by motions in an electrically-conducting medium, is another focal point of understanding solar magnetic activities [Parker, 1979]. In this section, close relations between dynamo effects and magnetic helicity are discussed.

\subsection{MHD Dynamo and Diamagnetic Dynamo}

A widely used scheme to discuss dynamo effects arising from MHD turbulence has been based on the mean-field electrodynamics [Krause and Rädler, 1980], where every quantity $x$ is divided into a mean part $\bar{x} \equiv<x>$, averaged over ensembles or space, 
and a turbulent part $\tilde{x}: x=\bar{x}+\widetilde{x}$. Therefore, the mean MHD Ohm's law can be written as,

$$
\overline{\mathbf{E}}+\overline{\mathbf{v}} \times \overline{\mathbf{B}}+<\widetilde{\mathbf{v}} \times \widetilde{\mathbf{B}}>=\eta \overline{\mathbf{j}},
$$

where the last term on the left-hand side is the mean electromotive force (EMF) $\mathcal{E}$ arising from turbulence, and it can be expressed approximately as

$$
\mathcal{E}=\alpha \overline{\mathbf{B}}-\beta \nabla \times \overline{\mathbf{B}}
$$

Here $\alpha$ and $\beta$ are determined by turbulence, often called the $\alpha$-effect and the $\beta$-effect [Parker, 1955]. It can be seen easily that the $\alpha$-effect, which has been regarded as an essential process for a working dynamo, represents an electromotive force generated by turbulence in the direction along the mean magnetic field.

In order to include other possible dynamo effects in a plasma, the same process can be repeated for the generalized Ohm's law (ignoring the electron inertial term) [Spitzer, 1962]

$$
\mathbf{E}+\mathbf{v} \times \mathbf{B}-\frac{\mathbf{j} \times \mathbf{B}}{e n}+\frac{\nabla P_{e}}{e n}=\eta \mathbf{j},
$$

where $n$ is the electron density and $P_{e}$ the electron pressure. Then the mean EMF in a turbulent plasma becomes

$$
\mathcal{E}=<\widetilde{\mathbf{v}} \times \widetilde{\mathbf{B}}>-<\tilde{\mathbf{j}} \times \widetilde{\mathbf{B}}>/ e \bar{n}
$$

where the second term is often called the Hall term and we have neglected $\left\langle\tilde{n} \nabla \widetilde{P}_{e}\right\rangle / e \bar{n}^{2}$ (a battery effect, see the next section $)$. Since $\mathbf{v}=\left(m_{i} \mathbf{v}_{i}+\right.$ $\left.m_{e} \mathbf{v}_{e}\right) /\left(m_{i}+m_{e}\right) \approx \mathbf{v}_{i}$ and $\mathbf{j}=e n\left(\mathbf{v}_{i}-\mathbf{v}_{e}\right)$, Eq.(12) can be rewritten as

$$
\mathcal{E}=<(\widetilde{\mathbf{v}}-\tilde{\mathbf{j}} / e \bar{n}) \times \widetilde{\mathbf{B}}>\approx<\widetilde{\mathbf{v}}_{e} \times \widetilde{\mathbf{B}}>,
$$

where $\mathbf{v}_{i}\left(\mathbf{v}_{e}\right)$ is the ion (electron) flow velocity. We note that the appearance of $\mathbf{v}_{e}$ only is consistent with the Ohm's law being a force balance of electrons.

The parallel component of $\mathcal{E}$, or the $\alpha$-effect, along the mean field are of interest. Therefore, only the perpendicular turbulent flow and magnetic field are relevant, i.e.,

$$
\mathcal{E}_{\|}=<\widetilde{\mathbf{v}}_{e} \times \widetilde{\mathbf{B}}>_{\|}=<\widetilde{\mathbf{v}}_{e \perp} \times \widetilde{\mathbf{B}}_{\perp}>.
$$

An alternative form of the parallel Ohm's law can be derived by substituting the perpendicular component of Eq.(11),

$$
\widetilde{\mathbf{v}}_{e \perp} \approx \tilde{\mathbf{v}}_{\perp}-\frac{\widetilde{\mathbf{j}}_{\perp}}{e \bar{n}} \approx \frac{\widetilde{\mathbf{E}}_{\perp} \times \overline{\mathbf{B}}}{\bar{B}^{2}}+\frac{\nabla_{\perp} \widetilde{P}_{e} \times \overline{\mathbf{B}}}{e \bar{n} \bar{B}^{2}},
$$

into Eq.(14) to yield

$$
\mathcal{E}_{\|}=<\widetilde{\mathbf{E}}_{\perp} \cdot \tilde{\mathbf{b}}_{\perp}>+<\nabla_{\perp} \widetilde{P}_{e} \cdot \tilde{\mathbf{b}}_{\perp}>/ e \bar{n}
$$

where $\mathbf{b} \equiv \mathbf{B} / B$.

We identify two possible $\alpha$-effects [ Ji et al., 1995, 1996] in Eq.(16). The first term $<\widetilde{\mathbf{E}}_{\perp} \cdot \widetilde{\mathbf{b}}_{\perp}>$, represents the contribution to $\widetilde{\mathbf{v}}_{e \perp}$ from the turbulent $\widetilde{\mathbf{E}}_{\perp} \times \overline{\mathbf{B}} / \bar{B}^{2}$ drift which is a MHD (single fluid) effect (MHD dynamo), while the second term, $<\nabla_{\perp} \widetilde{P}_{e} \cdot \tilde{\mathbf{b}}_{\perp}>/ e \bar{n}$, is the contribution from the turbulent electron diamagnetic drift $\nabla_{\perp} \widetilde{P}_{e} \times \overline{\mathbf{B}} / \bar{B}^{2}$ which is an electron fluid effect in the two-fluid framework (diamagnetic dynamo). We emphasize here that only the MHD dynamo effect has been studied in most dynamo theories and simulations, while both MHD and diamagnetic dynamo effects have actually been detected in the laboratory [Ji et al., 1995, 1996].

\subsection{Helicity Conservation During Dynamo Action}

Both the MHD and diamagnetic dynamos drive a parallel current twisting up the field lines, which can be translated into creation of magnetic helicity. Then one question may arise: can magnetic helicity be generated by dynamo action without any constraints? The answer is no: the total helicity must be conserved, except for a battery effect, as discussed below.

By using the generalized Ohm's law, the rate of change of helicity can be rewritten as

$$
\begin{aligned}
\frac{d K}{d t}= & -2 \int \eta \mathbf{j} \cdot \mathbf{B} d V-2 \int \frac{\nabla P_{e} \cdot \mathbf{B}}{e n} d V \\
& -\int\left(2 \phi \mathbf{B}+\mathbf{A} \times \frac{\partial \mathbf{A}}{\partial t}\right) \cdot d \mathbf{S}
\end{aligned}
$$

The first term on the RHS is not a dynamo effect but a resistive effect, which vanishes with zero resistivity. A finite resistivity introduces not only the usual resistive helicity decay but also a helicity increase or decrease during magnetic reconnection, a phenomenon which happens only in a resistive plasma (see Section 3 ). The last term on the RHS is surface integration, which transports helicity across space while conserving the total helicity. Indeed, the dynamo effect can originate from this surface term, as shall be seen in the next section.

The second term on the RHS of Eq.(17) can be rewritten as

$$
\int \frac{\nabla P_{e} \cdot \mathbf{B}}{e n} d V=\int \frac{T_{e}}{e} \mathbf{B} \cdot d \mathbf{S}+\int \frac{T_{e}}{e n} \nabla n \cdot \mathbf{B} d V
$$


where the first term is a surface term that does not change the total helicity while the second term does in certain conditions. Obviously, one such condition is a finite density gradient along the field line. However, this condition is not enough to change the total helicity. The integral of the second term in Eq.(18) is equivalent to

$$
\frac{T_{e}}{e n} \boldsymbol{\nabla} n \cdot \mathbf{B}=\boldsymbol{\nabla} \cdot\left(\frac{T_{e}}{e} \ln n \mathbf{B}\right)-\ln n \boldsymbol{\nabla} \frac{T_{e}}{e} \cdot \mathbf{B},
$$

where the first term leads to a surface term with no effects on the total helicity. From the second term, it can be seen that a finite temperature gradient is required for a finite change in the total helicity. Therefore, both finite gradients in density and electron temperature (of course also in electron pressure) along the field line are necessary conditions to change the total helicity. However, we note that such parallel gradients, especially $\nabla_{\|} T_{e}$, are very small owing to fast electron flow along the field lines. Such effects, often called the battery effect [Parker, 1979], provide only a seed for magnetic field to grow in a dynamo process and, of course, it can be accompanied by a small but finite magnetic helicity.

In summary, dynamo effects conserve the total helicity except for a small battery effect. This conclusion is consistent with the observation that the helicity is approximately conserved during magnetic relaxation [Ji, Prager, and Sarff, 1995]. In the following section, the battery effect will be ignored for simplicity.

\subsection{Helicity in Mean and Turbulent Fields}

Dynamo action amplifies a seed magnetic field into a large-scale, mean field and maintains it against resistive decay. Magnetic helicity associated with the mean fields must also be generated and maintained by dynamo effects. According to the last section, however, the helicity in the total (mean plus turbulent) field cannot be created (except for a small battery effect). Therefore, only two possibilities exist for the mean-field helicity: either it is transported across space or it is separated from the helicity associated with turbulent fields. We shall see that both mechanisms are possible depending on the nature of the dynamo process.

We begin with the rate of change of the helicity in the mean field, $K_{\mathrm{m}}=\int \overline{\mathbf{A}} \cdot \overline{\mathbf{B}} d V$, and the helicity in the turbulent field, $K_{\mathrm{t}}=\int<\widetilde{\mathbf{A}} \cdot \widetilde{\mathbf{B}}>d V$ :

$$
\begin{aligned}
\frac{d K_{\mathrm{m}}}{d t}= & -2 \int \overline{\mathbf{E}} \cdot \overline{\mathbf{B}} d V-\int\left(2 \bar{\phi} \overline{\mathbf{B}}+\overline{\mathbf{A}} \times \frac{\partial \overline{\mathbf{A}}}{\partial t}\right) \cdot d \mathbf{S}(20) \\
\frac{d K_{\mathrm{t}}}{d t}= & -2 \int<\tilde{\mathbf{E}} \cdot \widetilde{\mathbf{B}}>d V-\int(2<\tilde{\phi} \widetilde{\mathbf{B}}> \\
& \left.+<\tilde{\mathbf{A}} \times \frac{\partial \tilde{\mathbf{A}}}{\partial t}>\right) \cdot d \mathbf{S}
\end{aligned}
$$

and their sum, $K_{\mathrm{m}}+K_{\mathrm{t}}$, is the total mean helicity, $\bar{K}$. By using an alternative form of the generalized Ohm's law, $\mathbf{E}+\mathbf{v}_{e} \times \mathbf{B}+\nabla P_{e} / e n=\eta \mathbf{j}$, we have

$$
\begin{gathered}
\overline{\mathbf{E}}+\overline{\mathbf{v}}_{e} \times \overline{\mathbf{B}}+\frac{\nabla \bar{P}_{e}}{e n}+\mathcal{E}=\eta \overline{\mathbf{j}} \\
\widetilde{\mathbf{E}}+\widetilde{\mathbf{v}}_{e} \times \overline{\mathbf{B}}+\overline{\mathbf{v}}_{e} \times \widetilde{\mathbf{B}}+\widetilde{\mathbf{v}}_{e} \times \widetilde{\mathbf{B}}-\mathcal{E}+\frac{\nabla \widetilde{P}_{e}}{e n}=\eta \tilde{\mathbf{j}}
\end{gathered}
$$

where $\mathcal{E}=\left\langle\widetilde{\mathbf{v}}_{e} \times \widetilde{\mathbf{B}}>\right.$. Substituting these two equations into Eqs.(20) and (21), after some algebra, we obtain

$$
\begin{aligned}
\frac{d K_{\mathrm{m}}}{d t}= & -2 \int \eta \overline{\mathbf{j}} \cdot \overline{\mathbf{B}} d V+2 \int \mathcal{E} \cdot \overline{\mathbf{B}} d V \\
& -\int\left(2 \bar{\phi} \overline{\mathbf{B}}-2 \frac{\bar{P}_{e} \overline{\mathbf{B}}}{e n}+\overline{\mathbf{A}} \times \frac{\partial \overline{\mathbf{A}}}{\partial t}\right) \cdot d \mathbf{S} \\
\frac{d K_{\mathrm{t}}}{d t}= & -2 \int \eta<\tilde{\mathbf{j}} \cdot \widetilde{\mathbf{B}}>d V-2 \int \mathcal{E} \cdot \overline{\mathbf{B}} d V \\
& -\int<2 \tilde{\phi} \widetilde{\mathbf{B}}-2 \frac{\widetilde{P}_{e} \tilde{\mathbf{B}}}{e n}+\widetilde{\mathbf{A}} \times \frac{\partial \tilde{\mathbf{A}}}{\partial t}>\cdot d \mathbf{S} .
\end{aligned}
$$

The $\alpha$-effect appears as the second terms in the RHS of these equations but with opposite signs. It might be concluded that the dynamo effects would generate the same amount of helicity but with opposite signs in the mean field and the turbulent field [Seehafer, 1996]. However, it may not be the case depending on types of dynamo effects.

Following Eq.(16), the $\alpha$-effect is

$$
\begin{aligned}
\mathcal{E} \cdot \overline{\mathbf{B}}= & -<\nabla_{\perp} \tilde{\phi} \cdot \widetilde{\mathbf{B}}_{\perp}>-<\frac{\partial \tilde{\mathbf{A}}_{\perp}}{\partial t} \cdot \widetilde{\mathbf{B}}_{\perp}> \\
& +\frac{<\boldsymbol{\nabla}_{\perp} \widetilde{P}_{e} \cdot \widetilde{\mathbf{B}}_{\perp}>}{e n},
\end{aligned}
$$

where the three terms correspond to effects due to electrostatic, electromagnetic, and electron diamagnetic turbulence, respectively. Substituting Eq.(26) into Eqs.(24) and (25),

$$
\frac{d K_{\mathrm{m}}}{d t}=-2 \int\left(\eta \overline{\mathbf{j}} \cdot \overline{\mathbf{B}}+<\frac{\partial \tilde{\mathbf{A}}_{\perp}}{\partial t} \cdot \widetilde{\mathbf{B}}_{\perp}>\right) d V
$$




$$
\begin{aligned}
& -\int\left(2 \bar{\phi} \overline{\mathbf{B}}-2 \frac{\bar{P}_{e} \overline{\mathbf{B}}}{e n}+\overline{\mathbf{A}} \times \frac{\partial \overline{\mathbf{A}}}{\partial t}\right. \\
& \left.+2<\tilde{\phi} \widetilde{\mathbf{B}}-\frac{\widetilde{P}_{e} \widetilde{\mathbf{B}}}{e n}>\right) \cdot d \mathbf{S} \\
\frac{d K_{\mathrm{t}}}{d t}= & -2 \int\left(\eta<\tilde{\mathbf{j}} \cdot \tilde{\mathbf{B}}>-<\frac{\partial \tilde{\mathbf{A}}_{\perp}}{\partial t} \cdot \tilde{\mathbf{B}}_{\perp}>\right) d V \\
& -\int<\tilde{\mathbf{A}} \times \frac{\partial \tilde{\mathbf{A}}}{\partial t}>\cdot d \mathbf{S} .
\end{aligned}
$$

are obtained. In the case of electromagnetic turbulence, i.e., $\widetilde{\mathbf{v}}_{e}$ is driven by an inductive electric field, the dynamo effect generates the same amount of helicity both in the mean and turbulent fields but with opposite signs, as seen from the second terms of the above equations. Techniques often used in the laboratory to drive currents in a plasma by an incident electromagnetic wave fall into this category. The wave helicity is converted to the mean-field helicity by interaction between the wave and the background plasma. (We note that the last term in Eq.(28) represents an electromagnetic wave propagating across space without interacting with the mean field.) In the case of electrostatic or electron diamagnetic turbulence, i.e., $\widetilde{\mathbf{v}}_{e}$ is driven by electrostatic field or perpendicular electron pressure, the dynamo effect does not affect the turbulent helicity but merely transports the mean-field helicity across space, as seen from the surface terms in Eq.(27).

Therefore, it is crucial to know the type of turbulence which generates the dynamo effect in a turbulent plasma in order to assess the role of dynamo effects on magnetic helicity, even though the total helicity is always conserved. In the case of a laboratory plasma (the MST RFP), direct measurements indicated that the turbulence is predominantly electrostatic, thus causing helicity transport in the mean field with no effects on the turbulent field. Figure 9 shows such an example of measured helicity flux caused by the electrostatic turbulence [Ji, Prager, and Sarff, 1995] together with the measured $\alpha$-effect [Ji et al., 1994].

\section{CONCLUSIONS}

Magnetic helicity is closely related to magnetic reconnection and dynamo effects, both of which involve changes in magnetic field topology. Recent laboratory experiments have provided opportunities to test, verify, and discover the relationships between them as summarized below.

When two plasmas are driven to merge, the helicity content of each plasma determines the recon- necting angle which strongly affects the reconnection rate and the shape of the diffusion region. Conversely, magnetic reconnection events also strongly affect the global helicity, resulting in efficient helicity cancellation (but not dissipation) during counter-helicity reconnection and a finite helicity increase or decrease (but less efficiently than dissipation of magnetic energy) during co-helicity reconnection.

The turbulent electromotive force along the mean magnetic field ( $\alpha$-effect), due to either electrostatic turbulence or the electron diamagnetic effect, have been measured in an RFP plasma. These dynamo effects transport mean-field helicity across space without dissipation, as seen in direct measurements of helicity flux. When the dynamo effect is driven by electromagnetic turbulence, helicity in the turbulent field is converted to mean-field helicity. In all cases, however, dynamo processes conserve total helicity except for a small battery effect, consistent with the observation that the helicity is approximately conserved during magnetic relaxation.

Acknowledgments. The author is grateful to Drs. M. Yamada, R. Kulsrud, S. Hsu, and S. Prager for their collaborations.

\section{References}

Akasofu, S.-I., Physics of Magnetospheric Substorms, p.12, D. Reidel, Norwood, Mass., 1977.

Berger, M.A., Rigorous new limits on magnetic helicity dissipation in the solar corona, Geophys. Astrophys. Fluid Dyn., 30, 79, 1984.

Berger, M.A., and G.B. Field, The topological properties of magnetic helicity, J. Fluid Mech., 147, 133, 1984.

Biskamp, D., Magnetic reconnection, Phys. Rep., 237, $179,1994$.

Biskamp, D., Current sheet profiles in two-dimensional magnetohydrodynamics, Phys. Fluids B, 5, 3893, 1993.

Hegna, C.C., Self-consistent mean-field forces in turbulent plasmas: Current and momentum relaxation, Phys. Plasmas, 5, 2257, 1998.

Ji, H., A.F. Almagri, S.C. Prager, J.S.Sarff, Time-resolved observation of discrete and continuous MHD dynamo in the reversed-field pinch edge, Phys. Rev. Lett., 73, 668, 1994.

Ji, H., S.C. Prager, J.S.Sarff, Conservation of magnetic helicity during plasma relaxation, Phys. Rev. Lett., 74, 2945, 1995.

Ji, H., Y. Yagi, K. Hattori, A.F. Almagri, S.C. Prager, Y. Hirano, J.S. Sarff, T. Shimada, Y. Maejima, and K. Hayase, Effect of Collisionality and Diamagnetism on the Plasma Dynamo, Phys. Rev. Lett., 75, 1085, 1995.

Ji, H., S.C. Prager, A.F. Almagri, J.S. Sarff, Y. Yagi, 
Y. Hirano, K. Hattori, and H. Toyama, Measurement of the dynamo effect in a plasma, Phys. Plasmas, 3, 1935, 1996.

Ji, H., M. Yamada, S. Hsu, R. Kulsrud, Experimental test of the Sweet-Parker model of magnetic reconnection, Phys. Rev. Lett., 80, 3256, 1998.

Krause, F., and K.-H. Rädler, Mean-Field Magnetohydrodynamics and Dynamo Theory, Akademie-Verlag, Berlin, 1980.

Nagayama, Y., K.M. McGuire, M. Bitter, A. Cavallo, E.D. Fredrickson, K.W. Hill, H. Hsuan, A. Janos, W. Park, Analysis of sawtooth oscillations using simultaneous measurement of electron-cyclotron emission imaging and X-ray tomography on TFTR, Phys. Rev. Lett., 67, 3527, 1991.

Ono Y., A. Morita, M. Katsurai, M. Yamada, Experimental investigation of 3-dimensional magnetic reconnection by use of 2 colliding spheromaks, Phys. Fluids $B, 5,3691,1993$.

Parker, E.N., Hydromagnetic dynamo models, Astrophys. J., , 121, 293-314, 1955.

Parker, E.N., J. Geophys. Res., 62, 509, 1957.

Parker, E.N. Cosmical Magnetic Fields, 841 pp., Clarendon Press, Oxford, 1979

Petschek, H.E., Magnetic field annihilation, NASA Spec. Pub. SP-50, 425, 1964.

Pfister, H., and W. Gekelman, Demonstration of helicity conservation during magnetic reconnection using Christmas ribbons, Am. J. Phys., 59, 497, 1991.

Proctor, M.R.E., and A.D. Gilbert (Eds.), Lectures on Solar and Planetary Dynamos, 375 pp., Cambridge University Press, New York, 1994.

Rust, D.M., Spawning and shedding helical magnetic fields in the solar atmosphere, Geophys. Res. Lett., 21, 241, 1994.

Schnack, D.D., E.J. Caramana, R.A. Nebel, Three-dimensional magnetohydrodynamic studies of the reversed field pinch, Phys. Fluids, 28, 321, 1985.

Seehafer, N., Nature of the $\alpha$ effect in magnetohydrodynamics, Phys. Rev. E, 53, 1283, 1996.

Spitzer, L. Jr., Physics of Fully Ionized Gases (2nd Revised Edition), Interscience Publishers, New York, 1962.

Steinhauer, L.C., and A. Ishida, Relaxation of a twospecie magnetofluid, Phys. Rev. Lett., it 79, 3423, 1997; Relaxation of a two-species magnetofluid and application to finite-beta flowing plasmas, Phys. Plasmas, 5, 2609, 1998.

Stoneking, M.R., S.A. Hokin, S.C. Prager, G. Fiksel, H. Ji, D.J. Den Hartog, Particle transport due to magnetic fluctuations, Phys. Rev. Lett., 79, 549, 1994.

Sweet, P.A., and B. Lehnert (Eds.), Electromagnetic Phenomena in Cosmical Physics, 123pp., Cambridge University Press, New York, 1958.

Taylor, J.B., Relaxation of toroidal plasma and generation of reverse magnetic fields, Phys. Rev. Lett., 33, 1139, 1974 .
Taylor, J.B., Relaxation and Magnetic Reconnection in Plasmas, Rev. Mod. Phys., 58, 741, 1986.

Vasyliunas, V.M., Theoretical models of magnetic line merging, Rev. Geophys. Space Phys., 13, 303, 1975.

Woltjer, L., A theorem on force-free magnetic fields, Proc. Natl. Acad. Sci. USA, 44, 489, 1958.

Wright, A.N., and M.A. Berger, A physical description of magnetic helicity evolution in the presence of reconnection lines, J. Plasma Phys., 46, 179, 1991.

Yamada, M., Y. Ono, A. Hayakawa, M. Katsurai, Magnetic Reconnection of Plasma Toroids with Cohelicity and Counterhelicity, Phys. Rev. Lett., 65, 721, 1990.

Yamada, M., H. Ji, S. Hsu, T. Carter, R. Kulsrud, Y. Ono, F. Perkins, Identification of Y-Shaped and O-Shaped Diffusion Regions during Magnetic Reconnection in a Laboratory Plasma, Phys. Rev. Lett., 78, 3117, 1997.

Yamada, M., H. Ji, S. Hsu, T. Carter, R. Kulsrud, N. Bretz, F. Jobes, Y. Ono, F. Perkins, Study of Driven Magnetic Reconnection in a Laboratory Plasma, Phys. Plasmas 4, 1936, 1997.

H. Ji, Princeton Plasma Physics Laboratory, Princeton University, P.O. Box 451, Princeton, NJ 08543. (e-mail: hji@pppl.gov)

This preprint was prepared with AGU's LATEX macros v4. File pppl'report formatted October 6, 1998. 
(a) MRX setup

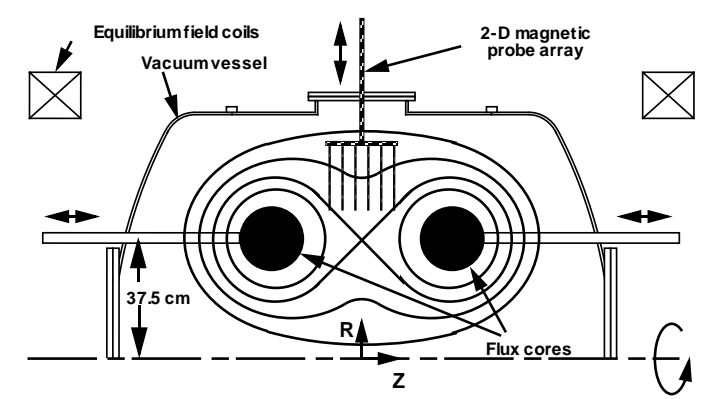

(b) "Pull" Reconnection

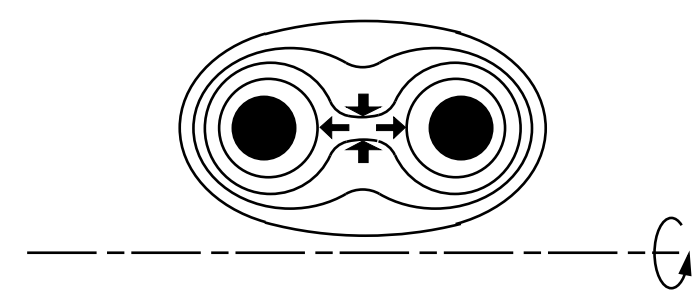

(c) "Push" Reconnection

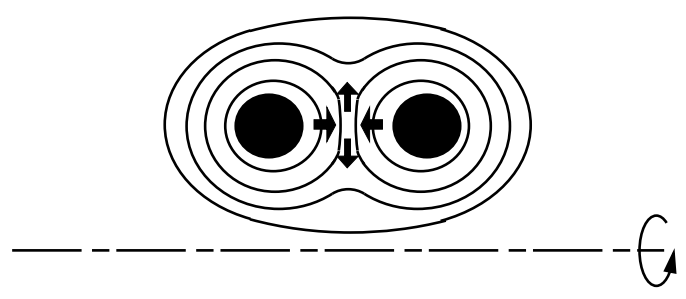

Figure 1. Experimental setup in MRX (a) and illustration of "push" (b) and "pull" (c) reconnection.

(a)
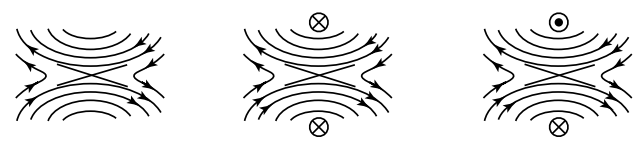

(b)
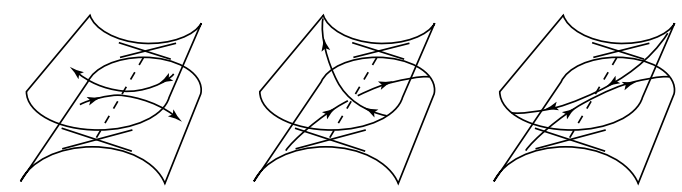

(i) Null-helicity

Reconnection

$\begin{array}{lll}\text { (ii) } & \text { Co-helicity (iii) } & \text { Counter-helicity } \\ \text { Reconnection } & \text { Reconnection }\end{array}$

Figure 2. (a) 2-D and (b) 3-D schematic views of magnetic reconnection for three cases: (i) null-helicity (ii) cohelicity and (iii) counter-helicity. 
(a) Null-helicity $(\mathrm{Bt}=0)$

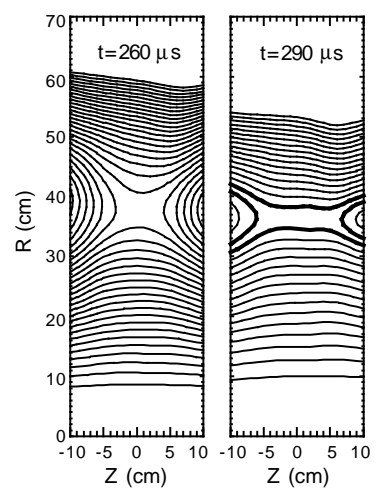

(b) Co-helicity $(\mathrm{Bt} \sim \mathrm{Bp})$

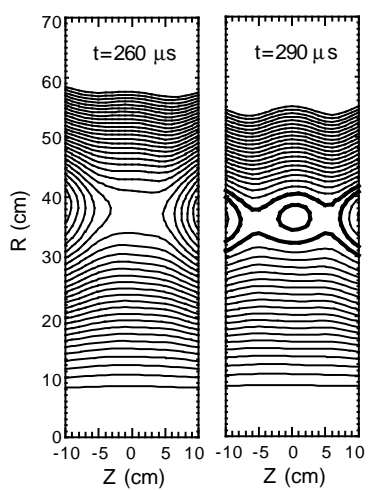

Figure 3. Time evolution of poloidal flux measured by internal magnetic probes By $t=290 \mu \mathrm{s}$, a double-Y shaped and O-shaped diffusion regions are formed in the (a) null-helicity case and (b) co-helicity case, respectively.

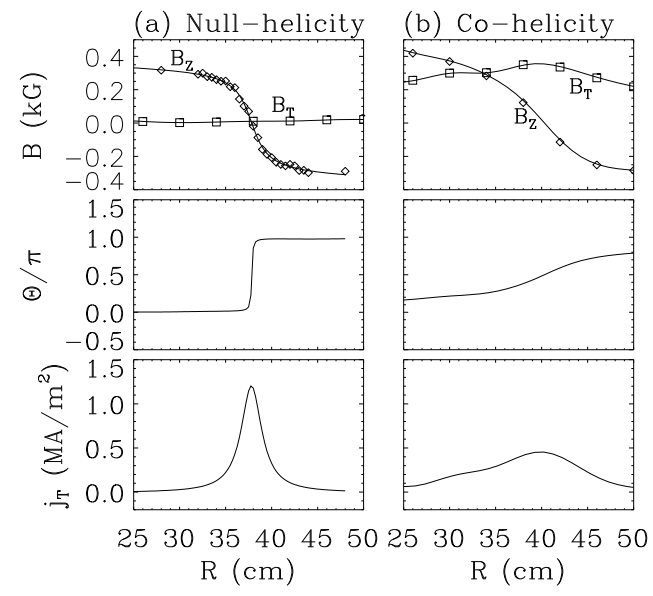

Figure 4. Radial profiles of measured $B_{Z}, B_{T}$, field line angle $(\Theta)$, and $j_{T}$ at $Z=0 \mathrm{~cm}$ and $t=290 \mu \mathrm{s}$ in the (a) null-helicity and (b) co-helicity cases. 


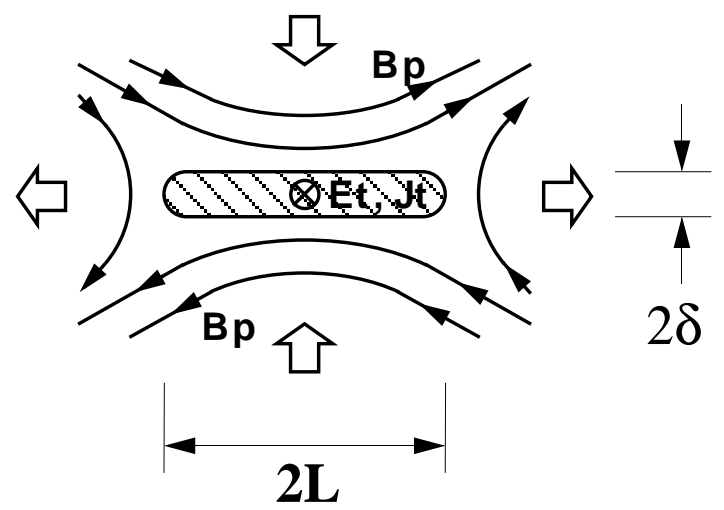

Figure 5. Illustration of a Sweet-Parker type of magnetic reconnection.

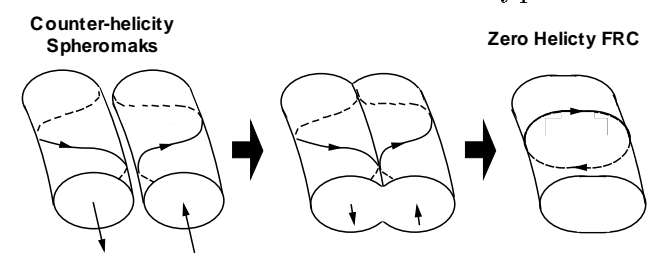

Figure 6. Helicity neutralization during a counter-helicity merging of spheromaks, resulting in a Field Reversed Configuration (FRC) with no magnetic helicity but finite ion kinetic helicity.

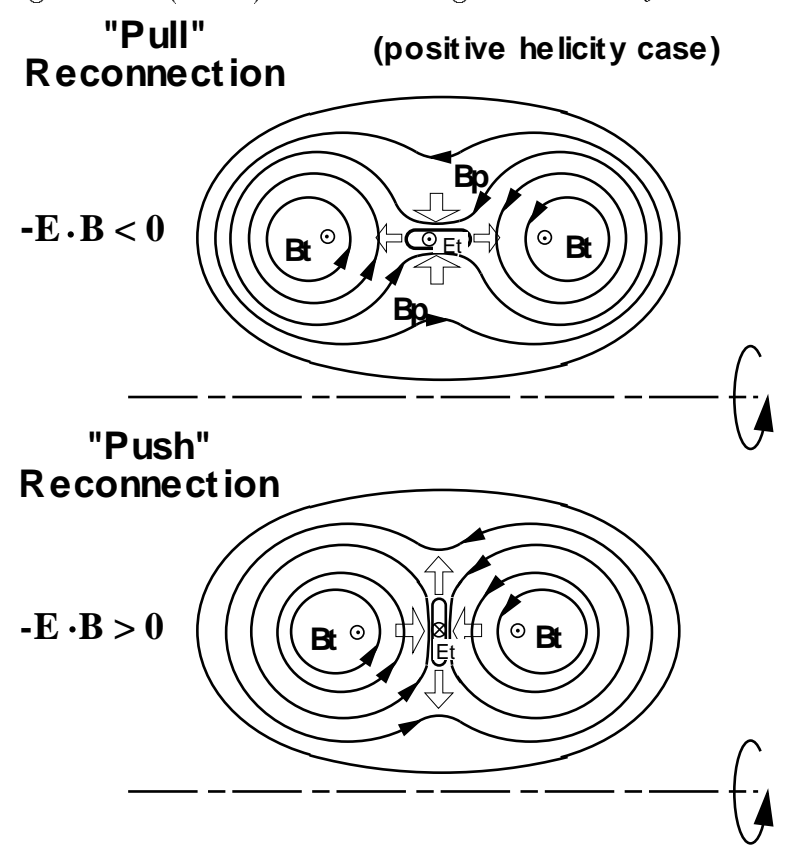

Figure 7. Illustration of co-helicity reconnection in the positive helicity case: "pull" ("push") reconnection leads to a decrease (an increase) in helicity. 

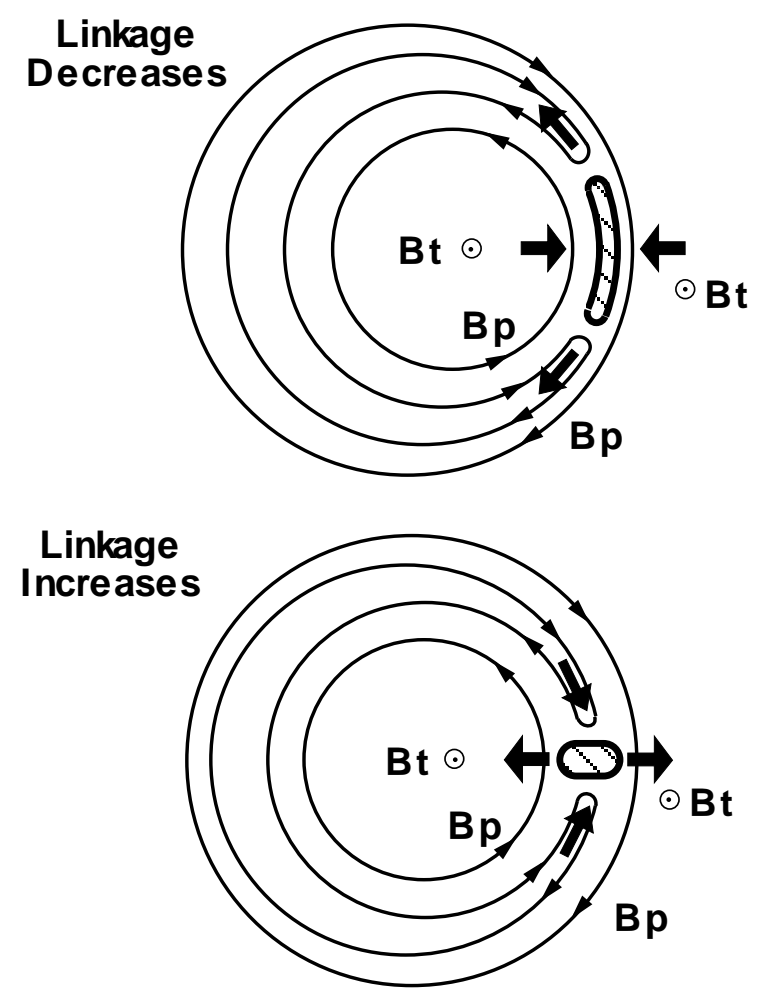

Figure 8. Two more examples for helicity change during co-helicity reconnection.
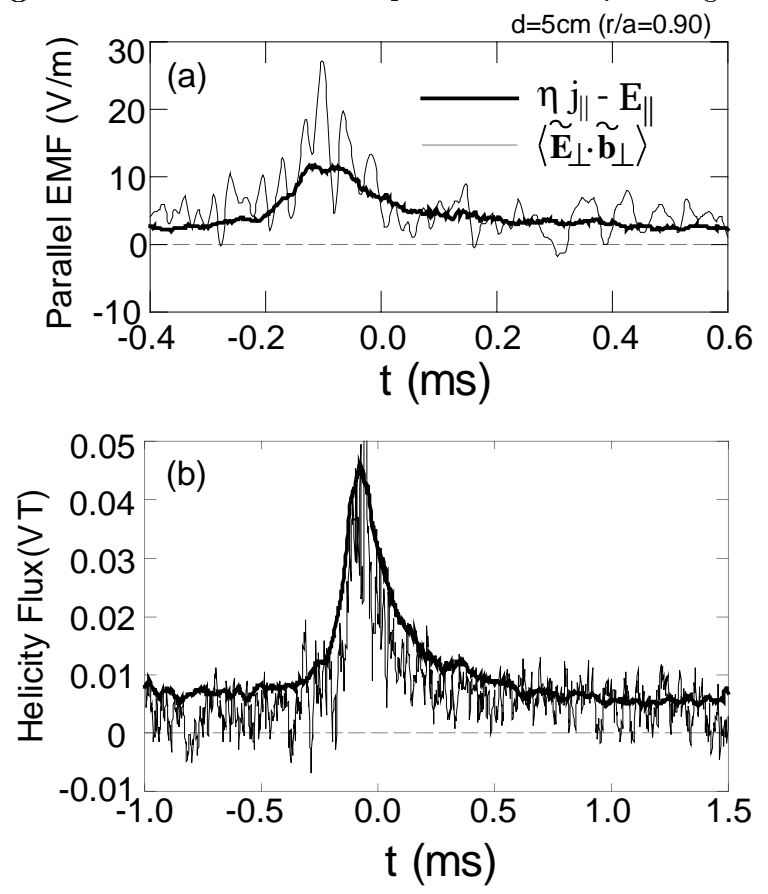

Figure 9. Measured (a) parallel EMF ( $\alpha$-effect) due to electrostatic turbulence, $\left\langle\widetilde{\mathbf{E}}_{\perp} \cdot \widetilde{\mathbf{b}}_{\perp}\right\rangle$, and (b) helicity flux (dotted line), $\langle\widetilde{\phi} \widetilde{\mathbf{B}}>$ in a laboratory plasma. The solid line in (b) is the prediction from the helicity balance equation. 